\title{
Toward Pleomorphic Reconfigurable Robots for Optimum Coverage
}

\author{
S. M. Bhagya P. Samarakoon (D), M. A. Viraj J. Muthugala $(\mathbb{D}$, Mohan R. Elara, \\ and Selva kumaran
}

Engineering Product Development Pillar, Singapore University of Technology and Design 487372, Singapore

Correspondence should be addressed to S. M. Bhagya P. Samarakoon; bhagya_samarakoon@mymail.sutd.edu.sg

Received 25 July 2020; Revised 8 December 2020; Accepted 8 January 2021; Published 21 January 2021

Academic Editor: Anirban Chakraborti

Copyright (C) 2021 S. M. Bhagya P. Samarakoon et al. This is an open access article distributed under the Creative Commons Attribution License, which permits unrestricted use, distribution, and reproduction in any medium, provided the original work is properly cited.

\begin{abstract}
Buildings are constructed for accommodating living and industrial needs. Floor cleaning robots have been developed to cater to the demand of these buildings. Area coverage and coverage time are crucial performance factors of a floor cleaning robot. Reconfigurable tiling robots have been introduced over fixed shape robots to improve area coverage in floor cleaning applications compared to robots with fixed morphologies. However, area coverage and coverage time of a tiling robot compromised one another. This study proposes a novel concept that considers the ability of a tiling robot to configure both its morphology and size according to the environment. This concept is inspired by the pleomorphism that could be seen in bacteria. In this regard, P-hTetro, a pleomorphic tiling robot that can reconfigure its morphology and size, is considered. A novel coverage strategy for realizing the size reconfiguration is also proposed. According to this strategy, the robot covers obstacle-free areas with its maximum size, while an obstacle cluster is covered after shrinking to an optimum size. The optimum size for reconfiguration is determined by the genetic algorithm based on the arrangement of the environment. The performance and behavior of the proposed P-hTetro have been compared against that of an existing tiling robot which has a fixed size. According to the statistical outcomes, a tiling robot with the ability to reconfigure its size can significantly improve the performance in the aspects of area coverage and coverage time compared to a tiling robot with no ability to reconfigure its size.
\end{abstract}

\section{Introduction}

Buildings are extensively constructed to accommodate the demand in living and industrial spaces due to socioeconomic requirements [1]. These living spaces need routine cleaning to uphold the living standard. Conventional cleaning methods of built environment demand extensive human labor, where cost, efficiency, and safety are major shortcomings [2]. Therefore, deployments of cleaning robots in application domains such as floor cleaning [3], wall cleaning [4], window cleaning [5], and garden cleaning [6] have been exercised to resolve the deficiencies of the conventional cleaning methods that demand extensive human labor.

Floor cleaning is one of the primary focuses among these application domains of cleaning robots, since it is a more frequent routine task in a building. Much effort has been made to improve the functionalities of floor cleaning robots to expand their productivity. For example, much work on improving the efficiency and safety of navigation through advanced path planning algorithms and sensing methods could be observed [7-10]. Many dirt detection and sensing algorithms have also been developed $[11,12]$. Furthermore, the use of multiple robots to improve the productivity of floor cleaning has been studied $[13,14]$. Apart from these core functionalities, work on improving aspects such as human-robot interaction could also be found in the literature $[15,16]$. Nevertheless, the majority of the methods discussed above have been proposed for robots with fixed morphologies.

Area coverage, energy usage, and coverage time are the crucial parameters that determine the productivity of a floor cleaning robot $[17,18]$. However, the area coverage performance of floor cleaning robots with a fixed morphology is limited in typical indoor environments due to the inclusion 
of objects with complex shapes such as furniture. It has been proven that a robot's ability to reconfiguration is highly beneficial in accessing narrow and confined spaces in other application domains such as rescue and exploration [19-21]. Similarly, self-reconfigurable robots have been introduced to cope with the area coverage problem faced by robots with fixed morphologies [22]. In this regard, various designs of reconfigurable robots intended for floor cleaning have been proposed. hTetro [23], hTetrakis [24], hTetran [25], and h-Trihex [26] can be considered as examples. A reconfigurable robot could access narrow spaces to a greater extent due to its ability to reconfigure into different morphologies. Hence, they can outperform the robots with a fixed shape in terms of area coverage. These robots utilize tiling strategies for planning the area coverage. Hence, these robots are often referred to as tiling robots.

Diverse aspects of this class of tiling robots have been studied to improve their productivity in floor cleaning applications. In this regard, much work on the realization of complete area coverage through efficient path planning through evolutionary algorithms and machine learning has been investigated $[24,27]$. Low-level controlling functionalities of robots, such as path tracking abilities, have also been extensively studied [26, 27]. Furthermore, reconfiguration strategies for improving the area coverage of tiling robots have been proposed $[28,29]$. With the aid of developments discussed above, reconfigurable tiling robots have proven their versatility in floor cleaning applications. Nevertheless, existing reconfigurable robots are merely capable of reconfiguring to a new shape while maintaining the original footprint area of the robot. Moreover, existing tiling robots are not capable of adjusting their total footprint area during reconfigurations.

A cleaning robot's ability to reconfigurations of both morphology and size of a robot would be beneficial in improving the performance while ascertaining the coverage performance. For example, the time taken for cleaning a given environment could be reduced when using a tiling robot with a comparatively larger size compared to a smaller size robot. On the contrary, the area coverage performance would be compromised when the size is large, since the accessibility of narrow spaces becomes impossible. If a robot can adapt its size in accordance with the environment, it could improve performance in terms of cleaning time while maintaining the same area coverage performance that would be expected from a smaller counterpart.

This study proposes a novel concept which considers that a tiling robot could reconfigure both morphology and size for improving the performance in terms of cleaning time and area coverage. Moreover, the work proposed in this study extends the abilities of the existing tiling robot to realize the pleomorphism. Pleomorphism is the ability of some microorganisms such as bacteria to change their morphology and size in accordance with the environment [30]. A bacterium that exhibits pleomorphisms is known as a pleomorphic bacterium. Hence, the proposed concept is named as pleomorphic-hTetro (P-hTetro). It is considered that $\mathrm{P}$-hTetro is capable of reconfiguring its size in addition to reconfiguring into different shapes by the existing hTetro.
The overview of pleomorphic-hTetro is discussed in Section 2. Area coverage methodology is presented in Section 3. Details on the validation of the proposed method are discussed in Section 4. Section 5 presents the concluding remarks.

\section{Overview of Pleomorphic-hTetro}

P-hTetro has four square shape blocks that are serially connected with hinges. It can reconfigure into seven distinct morphologies similar to the exiting reconfigurable tiling robot hTetro $[23,27,31]$, as depicted in Figure 1. The reconfiguration into different morphologies is facilitated by the hinges placed between blocks. P-hTetro has the ability to expand/shrink the size of each block, as depicted in Figure 2. Both length and width are simultaneously expanded/shrunk to maintain the square shape of each block. It is also assumed that the size reconfiguration actions of the blocks are synchronized. The lower bound and upper bound of the size reconfiguration are defined as $L_{L}$ and $L_{U}$. P-hTetro can reconfigure its size into any $L$ such that $L \in\left[L_{L}, L_{U}\right]$. In contrast, the existing tiling robot, hTetro, could not perform such expansion or shrinking action. As a result of the addition of this feature, P-hTetro can exhibit pleomorphism, where it can change both morphology and size. Incorporation of the ability to change the size of the robot through expanding action is the novel feature of P-hTetro with respect to state of the art. It is expected to have cleaning modules in all the blocks. The cleaning modules are made such a way that they can adapt as per the size of a block. P-hTetro can locomote with the aid of drive mechanisms attached to each block similar to the existing hTetro $[23,27,31]$. Perceiving the environment is expected to be accomplished through a Lidar. Moreover, all major functionalities of P-hTetro (other than size reconfiguration) are expected to be the same as hTetro.

The usefulness of the pleomorphism is explained with the aid of the situation depicted in Figure 3. The coverage time of a given environment can be reduced by using a robot with a larger size compared to a smaller robot. In contrast, the area covered would be limited due to the inaccessibility of narrow spaces due to its size constraint. This undesired compromising of area coverage could be avoided if the robot could shrink its size when it reaches confined areas. An explanatory video on this behavior is provided as a supplementary material. Therefore, P-hTetro could improve productivity since it could reduce the coverage time while ascertaining the level of area coverage by adapting both size and morphology in accordance with the environment setting.

\section{Area Coverage Strategy}

3.1. Determining Optimum Block Size. The main intentions of the p-hTetro are to maximize the area coverage and reduce the time for coverage. The example scenario depicted in Figure 4(a) is used to explain the area coverage strategy of the proposed P-hTetro. A metric map corresponding to a given environment is created through Lidar measurements, 


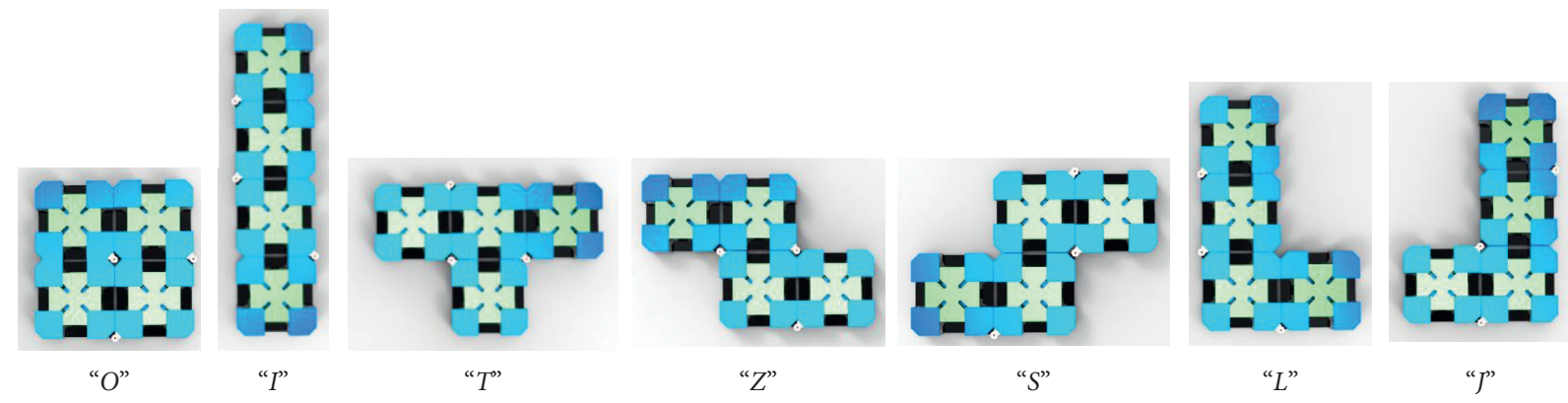

FIgURE 1: Seven distinct morphologies considered by P-hTetro for reconfiguration.

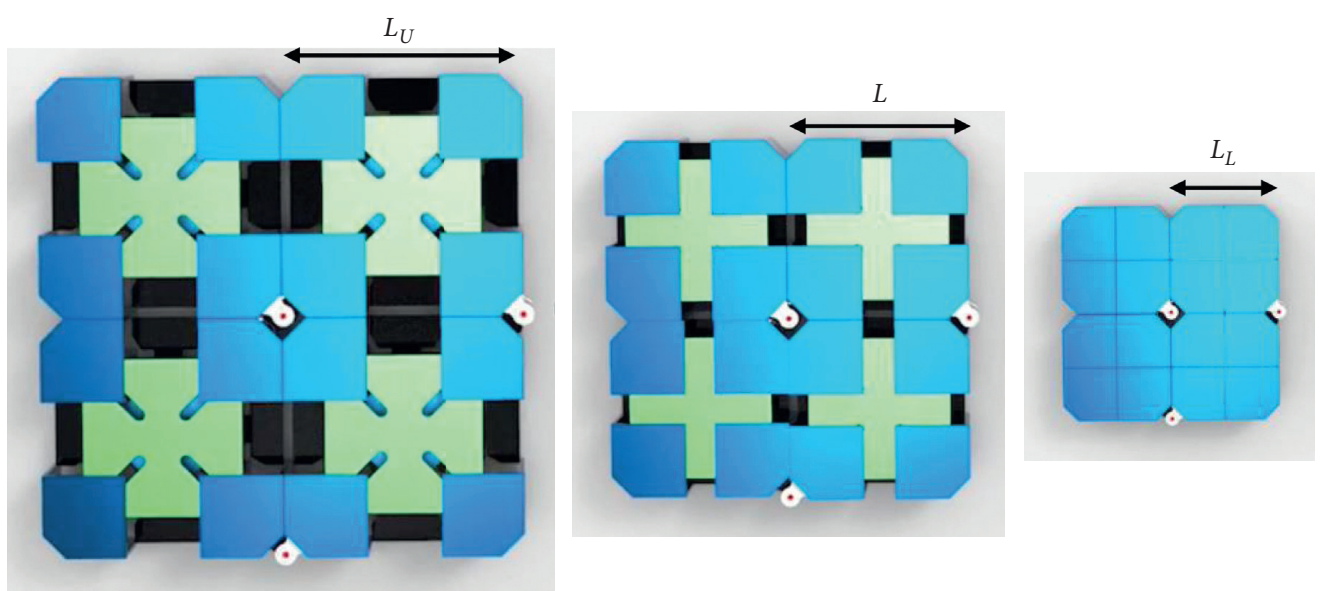

Figure 2: Size reconfiguration of P-hTetro. Here, the size reconfiguration is depicted considering "O" morphology. It should be noted that the size can be reconfigured in any morphology.

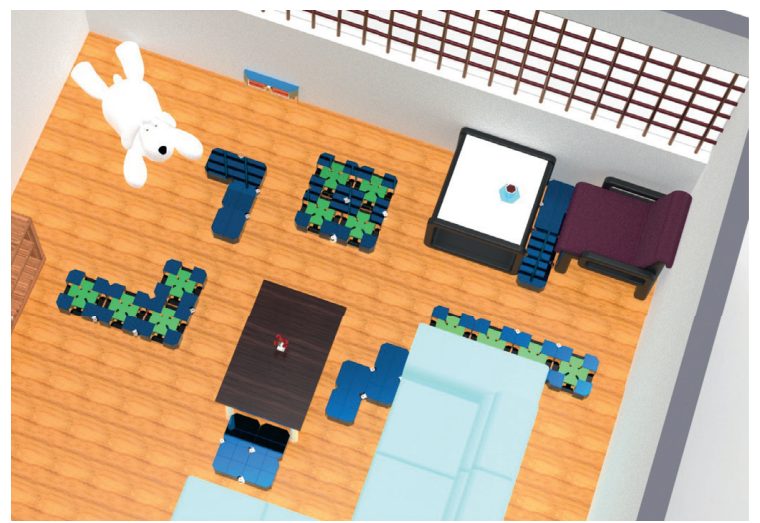

Figure 3: Operation of P-hTetro in a typical indoor environment. The robot shrinks its size in narrow spaces to improve the coverage. Similarly, the robot expands its size in free areas.

as depicted in Figure 4(b). Then, the metric map is converted to a grid map. The maximum size of the robot can cover a given space with less time than the smaller size case, since fewer cells lead the robot to less navigation to cover the grid. Thus, the size of a grid cell is selected as the maximum possible block size of P-hTetro $\left(L_{U}\right)$. A grid cell that is fully or partially occupied is considered as an obstacle area. The resultant occupancy grid map of the considered example scenario is depicted in Figure 4(c). If the robot performs the actions for area coverage solely considering this grid map, a vast area is left uncovered since the robot cannot access the confined area due to its size constraint. The size of the robot is adapted in accordance with the surrounding environment in addition to the reconfiguration of morphology to enhance the area coverage. In this regard, the clusters of grid cells tagged as obstacles in the main grid map (i.e., cell size $=L_{U}$ ) are identified and considered separately for the coverage strategy. For the obstacle cluster coverage, the robot should shrink its size $L \in\left[L_{L}, L_{U}\right]$ in such a way that it improves the productivity in terms of area coverage and coverage time.

The segments of the metric map corresponding to the identified obstacle clusters are considered in the process of obstacle cluster coverage. The optimum size of p-hTetro that maximize the area coverage while reducing the coverage time is determined for each obstacle cluster. An example segment of an obstacle cluster is given in Figure 4(d). In this regard, the optimum grid cell size for obstacle cluster coverage is determined by analyzing the environment. It should be noted that the robot's size and the cell size are the same.

The optimum cell size $L_{\text {opt }} \in\left[L_{L}, L_{U}\right]$ should be determined to ascertain the area coverage while lowering the number of grid cells for the navigation since coverage time increases with the number of cells to be covered. The placing of grid structure (i.e., determination of initial coordinate $\left.\left(x_{0}, y_{0}\right)\right)$ is an additional concern for improving the area 


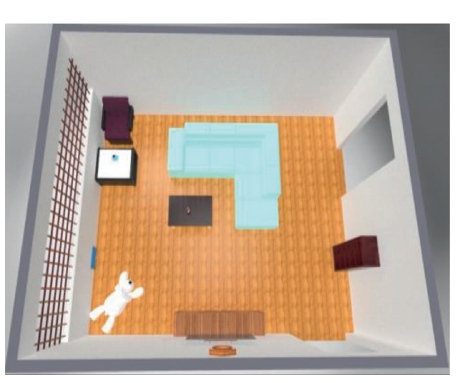

(a)

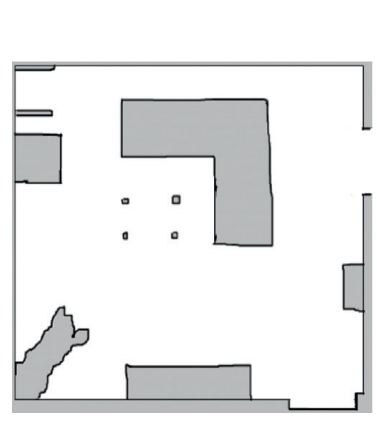

(b)

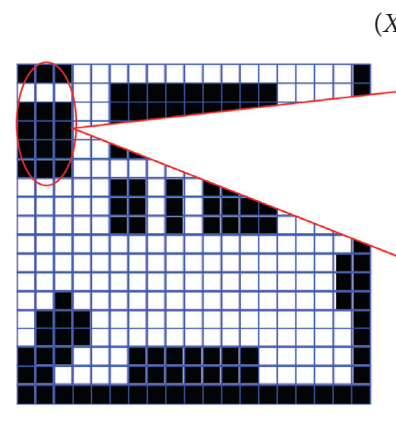

(c)

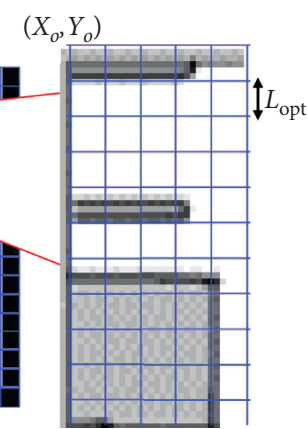

(d)

Figure 4: Explanation of the method proposed for determining the optimum block size. (a) Environment to be covered. (b) Metric map of the environment. (c) Occupancy grid map when cell size is $L_{U}$. (d) Segmented metric and placement of grid for coverage of the obstacle cluster.

coverage. Moreover, the robot needs to move the grids with different cell sizes on the segment of the metric map to find out the optimum cell size and grid placement that satisfies the required level of performance. Nevertheless, this is not a straightforward task due to the existence of many possible combinations of cell size and grid placement, and evaluation of all the combinations cannot be performed. Therefore, genetic algorithm (GA) is used to cope with this conundrum of finding the optimum cell size that improves area coverage while reducing the coverage time.

Much work has exploited the abilities of GA in solving this sort of optimization conundrum [32-34]. GA is a metaheuristic searching process inspired by natural evolution [35]. According to the expectation of the coverage strategy, the critical requirement is to maximize the area coverage (i.e., the total area of accessible grid cells) and minimize the number of cells in the grid. Therefore, the function given in (1) is used as the fitness function. Here, the number of cells that can be accessible for covering (i.e., nonobstacle) is denoted as $n$, and the length of the cell/robot block is symbolized as $L_{i}$. Thus, the total accessible area for coverage is represented by $n L_{i}^{2}$. K is a positive scalar constant that determines the tradeoff between the area coverage and coverage time. If $K$ is increased, the algorithm is more attempted to reduce the coverage time than improving the area coverage. $X_{i}$ and $Y_{i}$ are the coordinates of the initial position of the grid in a particular instance of GA. The flow of GA is given in Algorithm 1. Segmented metric map is fed to the algorithm as the input. The optimum size of the robot's block (i.e., $L_{\text {opt }}$ ) and the appropriate initial position of the grid $\left(x_{o}\right)$ are the outputs of the algorithm.

$$
f\left(X_{i}, Y_{i}, L_{i}\right)=-n L_{i}^{2}+K n \text {. }
$$

3.2. Overall Sequence of Operation. The overall sequence of operation of the robot during a coverage task is given in Algorithm 2. Initially, the metric map of the environment to be covered should be provided. The occupancy grid map of the environment is created considering a cell size equivalent to $L_{U}$. Optimum cell size (i.e., $L_{\text {opt }}$ ) for each obstacle cluster is determined through Algorithm 1, considering the corresponding segment of the metric map. The occupancy grid map of the obstacle cluster is then created. This process is repeated for all the obstacle clusters. During the coverage, when the robot encounters an obstacle cluster, the size of the robot is adapted based on the optimum size determined by the method in this study. After reconfiguring to an optimum size corresponding to an obstacle cluster, the robot's operation is analogous to a conventional tiling robot (which can only reconfigure the shape, not the size). Therefore, the coverage methods proposed for the conventional tiling robots could be used to determine an optimum navigation path and morphology reconfiguration to cover an occupancy grid map resulted from an obstacle cluster. The coverage method proposed in [27] for the conventional tiling robot has been proven to be efficient in covering a given occupancy grid map. Therefore, the navigation path and morphology reconfiguration to cover the created occupancy grid maps are expected to be conducted with the aid of the method proposed in [27]. This overall process is conducted offline, and the finalized coverage strategy is fed to the robot for the execution.

\section{Results and Discussion}

4.1. Simulation Setup. This performance and behavior of the proposed concept of P-hTetro have been evaluated through simulations conducted on MATLAB. In this regard, the proposed $\mathrm{P}$-hTetro has been compared against hTetro, which could only reconfigure its morphology, to assess the behavioral and performance rewarded from the ability of size reconfiguration. It was considered that the lower and upper bounds of size reconfiguration (i.e., $L_{L}$ and $L_{U}$ ) are $20 \mathrm{~cm}$ and $40 \mathrm{~cm}$, respectively. The parameters of GA have been configured to the following settings for the simulations. The population size was taken as 50, since the number of variables is three. The selection method of the GA was the stochastic uniform approach, and rank scaling function was used for evaluating the selection of best fits. The crossover fraction and elite fractions are configured to 0.8 and 0.05 , respectively.

Fifteen heterogenous obstacle clusters that can be observed from typical indoor environments have been 


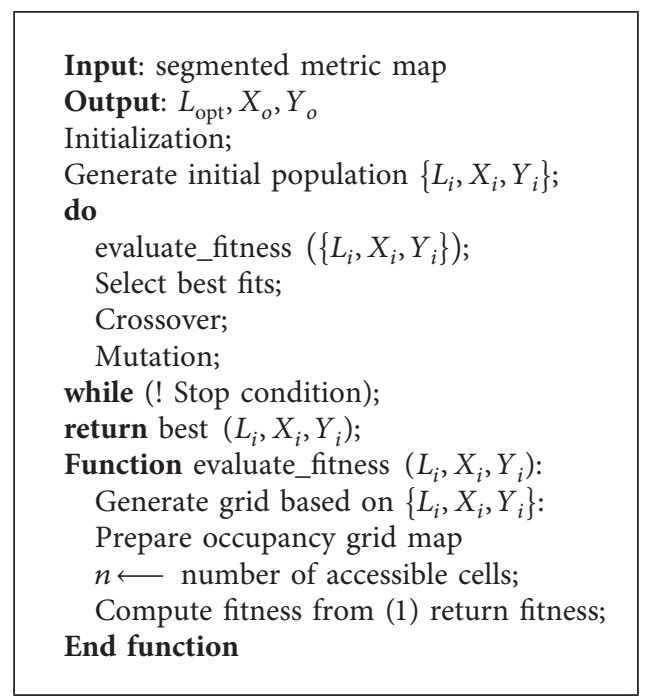

Algorithm 1: GA for determining Robot's block size.

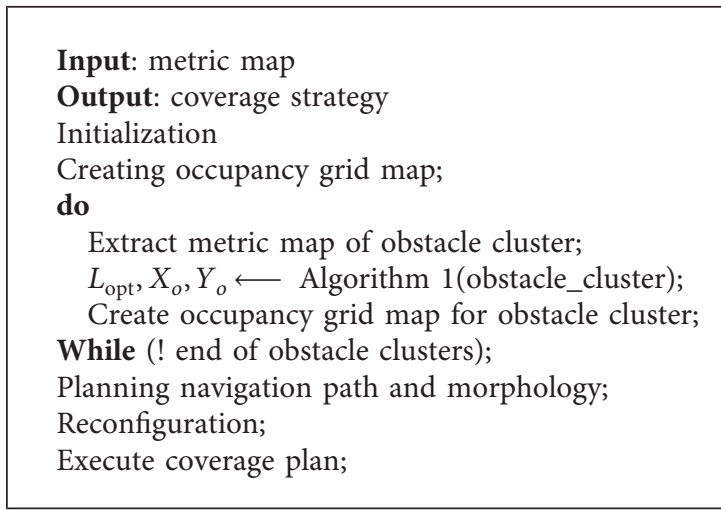

Algorithm 2: Overall operation.

considered for the simulations. The proposed strategy for the area coverage of P-hTetro has been tested on these test cases, and the uncovered area $\left(A_{U}\right)$ and the number of cells for coverage $(n)$ in each case have been considered as the performance indicators. $A_{U}$ and $n$ resulted from a robot with no ability to reconfigure its size (i.e., abilities similar to hTetro $[23,27,31])$ have also been evaluated for each test case as the benchmarking baselines. Moreover, the size of the robot was considered as fixed. hTetro fixed with the sizes $L_{U}$ and $L_{L}$ have been separately examined in this regard. The arrangements of five test cases, along with the resulted grids, are depicted in Figure 5 as sample cases. The key performance parameters corresponding to the sample test cases are given in Table 1.

4.2. Results. The arrangement of the environment in the test scenario "a" is depicted in Figure 5(a). An obstacle cluster resulted from a typical arrangement of a bedroom consisted of a bed, a table, and a chair is considered here. An uncovered area (i.e., $A_{U}$ ) of $3.7 \mathrm{~m}^{2}$ resulted in the case of the robot with no ability of size reconfiguration (i.e., the size of
hTetro was fixed to $\left.L_{U}\right)$. In contrast, $A_{U}$ resulted from the case of P-hTetro was $1.72 \mathrm{~m}^{2}$. The resulted grid of this scenario is depicted in Figure 5(a), and the accessible cells are annotated on it. This grid consists of 44 accessible cells. In the case of the robot fixed with a size of $L_{L}, A_{U}$ of $1.42 \mathrm{~m}^{2}$ was observed. $A_{U}$ was less than the case of P-hTetro in this case. However, the robot needs to access 56 cells yielding to a higher coverage time than P-hTetro.

Similar behavior and performance were observed for all 15 test scenarios. The means of $A_{U}$ for the three robots during all the test scenarios are given in Figure 6(a). The lowest mean of $A_{U}$ was observed for the robot's case with a fixed size of $L_{L}\left(M=1.92 \mathrm{~m}^{2}, \mathrm{SD}=1.08\right)$, while the highest mean $A_{U}$ was observed for the robot fixed with the size of $L_{U}$ $\left(M=6.27 \mathrm{~m}^{2}, \mathrm{SD}=3.29\right)$. For the case of P-hTetro, the mean of $A_{U}$ was $2.32 \mathrm{~m}^{2}(\mathrm{SD}=1.18)$. A one-way ANOVA test has been conducted to evaluate the statistical significance of results. A one-way ANOVA test is a statistical technique used to scrutinize if the means of two or more groups are significantly different from each other. The results of the ANOVA test are given in Table 2. A significance level of 0.05 has been considered in this regard to maintain a type I error 


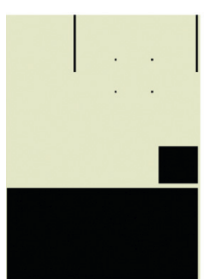

- P-hTetro - Fixed size $\left(L_{L}\right)$
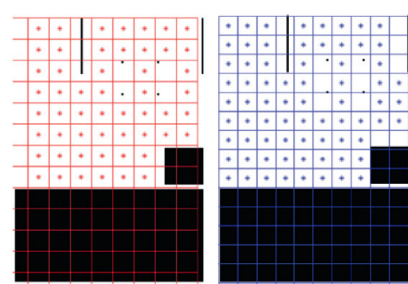

Footprint layer of the scenarios

$*, *$ Accessible cell

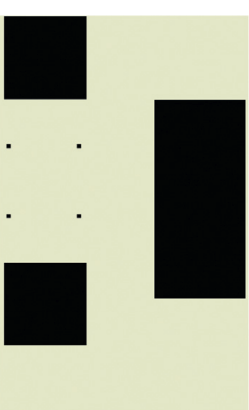

- P-hTetro

— Fixed size $\left(L_{L}\right)$

(a)

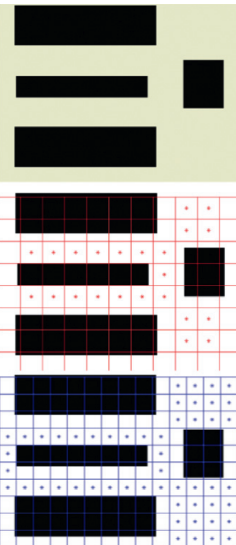

P-hTetro

Fixed size $\left(L_{L}\right)$

Footprint layer of

the scenarios

$*, *$ Accessible cell

(c)
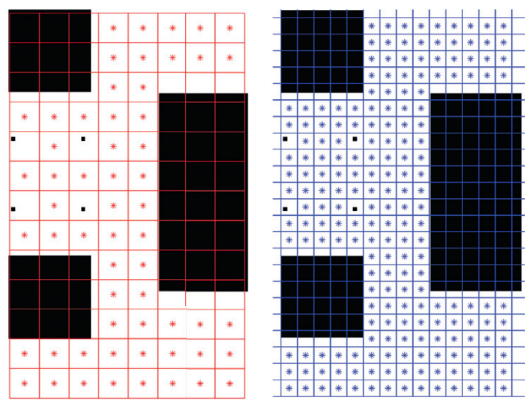

Footprint layer of the scenarios *,* Accessible cell

(b)

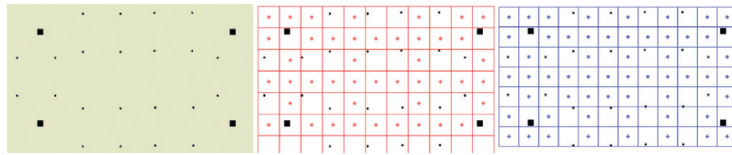

P-hTetro Fixed size $\left(L_{L}\right)$

Footprint layer of the scenarios

$*, *$ Accessible cell

(d)

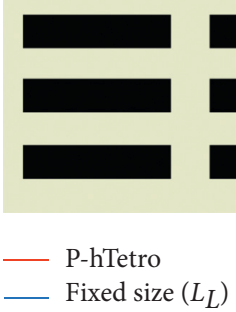

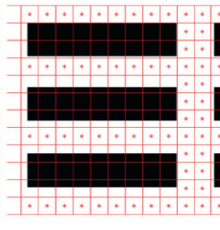

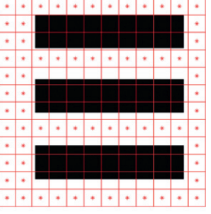

Footprint layer of the scenarios $*, *$ Accessible cell

Figure 5: Sample of test scenarios taken as obstacle clusters. In each case, the first figure depicts the footprint layer, the red-colored grid represents the optimum cell size generated for P-hTetro, and the grid resulting for the robot with fixed size $L_{L}$ is depicted in blue. (a) A segment of a bedroom. (b) and (c) A couch at a lobby or a living area. (d) Meeting room. (e) The seating arrangement of a waiting area.

TABLE 1: Sample results for comparison of area coverage and coverage time.

\begin{tabular}{|c|c|c|c|c|c|c|}
\hline \multirow{2}{*}{ Case } & \multicolumn{3}{|c|}{ GA } & \multicolumn{2}{|c|}{$L=L_{L}$} & \multirow{2}{*}{$\begin{array}{c}L=L_{U} \\
A_{U}\left(\mathrm{~m}^{2}\right)\end{array}$} \\
\hline & $L(\mathrm{~cm})$ & $A_{U}\left(\mathrm{~m}^{2}\right)$ & $n$ & $A_{U}\left(\mathrm{~m}^{2}\right)$ & $n$ & \\
\hline $\bar{a}$ & 21.2 & 1.72 & 44 & 1.47 & 56 & 3.7 \\
\hline$b$ & 26 & 2.21 & 23 & 1.69 & 52 & 3.77 \\
\hline$d$ & 23.8 & 4.15 & 108 & 3.63 & 166 & 10.27 \\
\hline$e$ & 22.5 & 0.95 & 53 & 0.75 & 72 & 3.64 \\
\hline$f$ & 35.8 & 2.55 & 58 & 2.07 & 198 & 9.99 \\
\hline
\end{tabular}




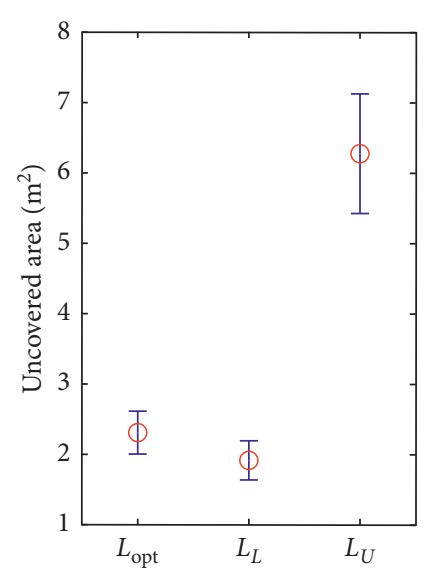

(a)

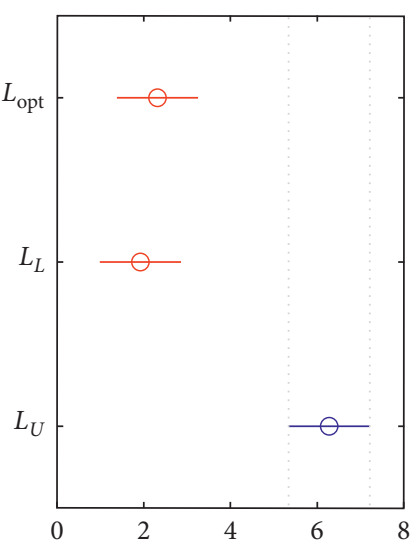

(b)

Figure 6: (a) Mean variation of uncovered area of P-hTetro (i.e., $L_{\text {opt }}$ ) and hTetro with fixed sizes of $L_{L}$ and $L_{U}$. The error bars represent the standard error of mean. (b) Comparison intervals of Tukey's honest significant difference criterion. It is considered that there is no significant difference between the means of corresponding groups if intervals of two groups overlap.

TABLE 2: Results of the ANOVA test on the uncovered area.

\begin{tabular}{lcccc}
\hline Source of variation & Sum of squares (SS) & Degrees of freedom (df) & Mean square (MS) & $F$ \\
\hline Robot configurations & 173.805 & 2 & 86.9023 & 19.52 \\
Error & 186.995 & 42 & 4.4523 & $1.013 \times 10^{-6}$ \\
Total & 360.8 & 44 & & \\
\hline
\end{tabular}

less than 5\%. According to the test statics, at least one mean is significantly different from others $\left(F_{2,42}=19.52, P \leq 0.001\right)$. Tukey's honest significant difference criterion was used as a post hoc test to evaluate the pairwise differences (results are given in Figure 6(b)). According to the post hoc test, the means of $A_{U}$ resulted from P-hTetro and hTetro of size $L_{L}$ were significantly less than that of hTetro of size $L_{U}$. There is no significant difference between P-hTetro and the robot with a fixed size of $L_{U}$ (hTetro of size $L_{L}$ ) in terms of $A_{U}$. Furthermore, the effect size has been evaluated considering Cohen's $d$ [36] that indicates the standardised difference between two means. The reduction of $A_{U}$ resulted from P-hTetro is noteworthy, since a very large effect size was observed according to Cohen's $d$ (Cohen's $d=1.78$; Cohen's $d>1.2$ is considered as a very large effect [36]). Moreover, these results confirm that P-hTetro can significantly improve the area coverage compared to hTetro with a fixed size of $L_{U}$ while ascertaining the same level of area coverage compared to hTetro with a fixed size of $L_{L}$.

The reduction of coverage time while ascertaining the area coverage is the main objective of $\mathrm{P}$-hTetro. The coverage time is dependent on the number of cells to be visited (i.e., $n$ ) to cover a given environment. The means of $n$ during the test scenarios are given in Figure 7, along with error bars. The mean $n$ of P-hTetro was $57.2(\mathrm{SD}=29.37)$, while the mean $n$ of hTetro of size $L_{L}$ was $108.8(\mathrm{SD}=63.27)$. A paired $t$-test has been conducted to evaluate the statistical significance of the two means. A paired $t$-test is statistical technique used to compare two means that are from the same individual, object, or related units. The results of the $t$-test are given in
Table 3. A significance level of 0.05 has been considered in this regard. According to the test statistics, P-hTetro needs to visit a significantly lesser number of cells than the case of hTetro of size $L_{L}\left(t_{14}=-4.12, P=0.001\right)$. Furthermore, this reduction is large, according to Cohen's $d$ (Cohen's $d=1.14$; Cohen's $d>0.8$ is considered as the large effect [36].). Moreover, these results confirm that the coverage time taken by P-hTetro is significantly lower than that of hTetro with a size of $L_{L}$.

Overall, the statistical conclusions on area coverage and coverage time concluded that the proposed P-hTetro is capable of significantly reducing coverage time compared to the same sort of robot fixed to a smaller size while ascertaining the same level of area coverage expected from the smaller robot. If a tiling robot is large (fixed), the performance in terms of area coverage is compromised for the performance improvement in terms of coverage time. On the other hand, if a tiling robot is small (fixed), the performance of coverage time is compromised to the area coverage. Nevertheless, the ability to adapt the size of a tiling robot avoid the shortcomings mentioned above. Moreover, significantly higher performance in terms of area coverage and coverage time can be observed from a tiling robot with the ability to reconfigure its size compared to a tiling robot with no ability to reconfigure its size.

4.3. Discussion. The existing tiling robots are not capable of reconfiguring their size. This study introduces the concept of pleomorphism to tiling robots. The ability of pleomorphism was inspired by the behavior of pleomorphic bacteria that 


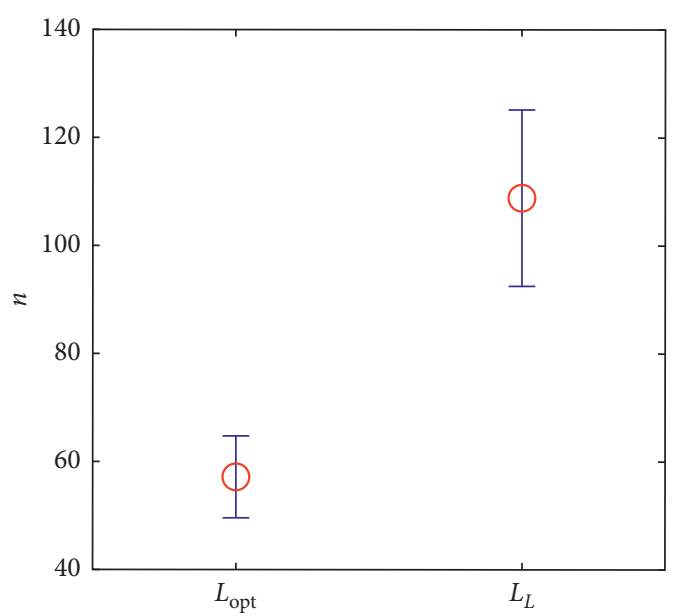

FIGURE 7: Variation of means of number of cells to visited in the cases of P-hTetro (i.e., $L_{\text {opt }}$ ) and hTetro with a fixed size of $L_{L}$ error bars. The error bars represent the standard error of mean.

TABle 3: Results of the $t$-test on number of cells to be visited.

\begin{tabular}{lccccc}
\hline $\begin{array}{l}\text { Robot } \\
\text { configuration }\end{array}$ & $\begin{array}{c}\text { Mean } \\
(M)\end{array}$ & $\begin{array}{c}\text { Standard } \\
\text { deviation } \\
(\mathrm{SD})\end{array}$ & $\begin{array}{c}\text { Degrees of } \\
\text { freedom } \\
(\mathrm{df})\end{array}$ & $t$ & $P$ \\
\hline$L_{\mathrm{opt}}$ & 57.2 & 29.37 & 14 & -4.12 & 0.001 \\
$L_{L}$ & 108.8 & 63.27 & 14 & & \\
\hline
\end{tabular}

can adapt morphology and size according to the environment. The pleomorphism concept enables a tiling robot to reconfigure its size apart from the morphology reconfiguration. A coverage strategy based on GA has been proposed to realize the pleomorphic behavior on a tiling robot by determining the optimum size for reconfiguration. These are the novel contributions of the work proposed in this study. According to the simulation results, the proposed coverage strategy can determine the optimum size for reconfiguring the size of a tiling robot in such a way that it improves the performance in terms of coverage time without compromising the area coverage.

Area coverage and coverage time are key performance indicators for a robot intended for floor cleaning [17]. However, the existing tiling robots intended for floor cleaning have to compromise area coverage and coverage time for one another. This shortcoming degrades the productivity of a tiling robot since they cannot reconfigure their size. The simulation outcomes confirm that a tiling robot with the ability to reconfigure its size can significantly improve the performance from the perspective of area coverage and coverage time compared to a tiling robot with no ability to reconfigure its size. Moreover, the proposed concept can eliminate the shortcoming of the existing reconfigurable tiling robot mentioned earlier. Therefore, it can be concluded that pleomorphism could significantly contribute to improving the productivity of reconfigurable tiling robots intended for floor cleaning applications.

The performance and behavior of the proposed concept of pleomorphism have been evaluated and discussed based on hTetro $[23,27,31]$, which is one of the versatile and widely used reconfigurable tiling robots. Even though the proposed concept is formulated based on hTetro, pleomorphism could be applied to other tiling robots. For example, the concept of pleomorphism could be applied to hTromino, which has only three square shape blocks. Further investigation on the generalization of the proposed concept for the class of reconfigurable tiling robots is proposed for future work.

The implications of this study have been concluded based on simulation results. These simulations have been conducted considering realistic parameters for robots and environments. For example, realistic settings of indoor environments such as bedrooms and waiting areas have been used in the simulation environment. Thus, the outcomes concluded based on the simulation results would not be withdrawn from real scenarios. Furthermore, the existing reconfigurable tiling robot can only reconfigure its shape while maintaining the same size. Moreover, the existing reconfigurable tiling robot cannot reconfigure their size. This study is the first study in a line of research that considers a tiling robot's ability to reconfigure its size apart from morphology reconfiguration. Moreover, this work could be lined up as proof of concept for this new direction (i.e., pleomorphism) for improving the abilities of reconfigurable tiling robots. Therefore, the implications concluded in this study are useful for making a substantial step toward the development of tiling robots. Real-world experiments with the proposed P-hTetro are expected to be performed in the next stage of the research.

According to [18], energy usage is another crucial factor determining the productivity of a floor cleaning robot apart from area coverage, and they are often conflicting entities. This study reports the initial formulation of this novel research niche, where a reconfigurable robot exhibits pleomorphism. Thus, the scope of this study is limited to improving the performance of a reconfigurable tiling robot in terms of area coverage and coverage time by considering the pleomorphism. The area coverage and energy usage could be appropriately balanced by utilizing the method proposed in [18]. The integration of such an energy tradeoff method with the P-hTetro is proposed for future work.

\section{Conclusion}

Socioeconomics complexity leads to the development of floor cleaning robots. Reconfigurable tiling robots have been introduced to overcome the shortcomings of robots with fixed morphologies. Area coverage and coverage time are key performance indicators that reflect the productivity of these robots. However, the existing tiling robot has a major shortcoming from improving performance in both area coverage and coverage time since those entities are conflicting with each other due to the nonadaptability of size.

This study proposed a novel concept, which considers that a tiling robot can reconfigure its size apart from the reconfiguration of its morphology to improve performance from the perspective of both area coverage and coverage time. This concept has been inspired by pleomorphism, which is the ability of some microorganisms to adapt size 
and morphology per environmental changes. A novel design of a reconfigurable, pleomorphic-hTetro (P-hTetro) that can reconfigure its morphology and size has been considered in this regard. Moreover, P-hTetro considers that the existing tiling robot, hTetro, can reconfigure its size in addition to morphology reconfiguration. Furthermore, a coverage strategy for P-hTetro has been proposed. This coverage strategy allows the optimum size reconfiguration of a tiling robot according to the environment setting to improve the performance. Genetic algorithm (GA) has been proposed to determine the robot's optimum block size based on the setting of an obstacle cluster.

Simulations were conducted considering typical settings of indoor spaces where possible obstacle clusters are presented. The simulation results validate the real-world applicability of the proposed concept. According to the statistical outcomes, the ability to reconfigure its size can significantly improve a tiling robot's performance in terms of area coverage and coverage time, which are key performance indicators of a floor cleaning robot. Therefore, it can be concluded that the novel concept proposed in this work contributed to the idea of realizing a perfect floor cleaning robot.

\section{Data Availability}

No data were used to support this study.

\section{Disclosure}

The funders had no role in the design of the study; in the collection, analyses, or interpretation of data; in the writing of the manuscript; or in the decision to publish the results.

\section{Conflicts of Interest}

The authors declare that they have no conflicts of interest.

\section{Acknowledgments}

This research was supported by the National Robotics Programme under its Robotics Enabling Capabilities and Technologies (Funding Agency Project no. 19225 00051) and National Robotics Programme under its Robot Domain Specific (Funding Agency Project no. 19222 00058) and administered by the Agency for Science, Technology, and Research.

\section{Supplementary Materials}

An explanatory video of the proposed P-hTetro is provided as a supplementary material. (Supplementary Materials)

\section{References}

[1] C. Thuesen and N. Koch-Ørvad, "Construction transformation," in Proceedings of the Sustain Conference 2018: Creating Technology for a Sustainable Society, Technical University of Denmark, Kongens Lyngby, Denmark, November 2018.

[2] J. Min, Y. Kim, S. Lee, T.-W. Jang, I. Kim, and J. Song, "The fourth industrial revolution and its impact on occupational health and safety, worker's compensation and labor conditions," Safety and Health at Work, vol. 10, no. 4, pp. 400-408, 2019.

[3] H.-Y. Park and J. Lee, "Quadruple-pad floor-mopping robot," International Journal of Precision Engineering and Manufacturing, vol. 21, no. 3, pp. 427-436, 2020.

[4] M. A. V. J. Muthugala, M. Vega-Heredia, R. E. Mohan, and S. R. Vishaal, "Design and control of a wall cleaning robot with adhesion-awareness," Symmetry, vol. 12, no. 1, p. 122, 2020.

[5] J. Hong, T. Kim, H. Chae et al., "Design of window-cleaning robotic manipulator with compliant adaptation capability," IEEE/ASME Transactions on Mechatronics, vol. 25, no. 4, 2020.

[6] J. Bai, S. Lian, Z. Liu, K. Wang, and D. Liu, "Deep learning based robot for automatically picking up garbage on the grass," IEEE Transactions on Consumer Electronics, vol. 64, no. 3, pp. 382-389, 2018.

[7] T. Sasaki, G. Enriquez, T. Miwa, and S. Hashimoto, "Adaptive path planning for cleaning robots considering dust distribution," Journal of Robotics and Mechatronics, vol. 30, no. 1, pp. 5-14, 2018.

[8] H.-Y. Huang, Y.-W. Hu, M.-F. Lu, S.-S. Chen, J.-T. Jeng, and W.-P. Chen, "An obstacle avoidance of large-scale indoor tricycle drive cleaning robot using laser scanner," in Proceedings of the 2017 Joint 17th World Congress of International Fuzzy Systems Association and 9th International Conference on Soft Computing and Intelligent Systems (IFSA-SCIS), pp. 1-6, IEEE, Otsu, Japan, June 2017.

[9] Z. Zhao, W. Chen, C. C. Peter, and X. Wu, "A novel navigation system for indoor cleaning robot," in Proceedings of the 2016 IEEE International Conference on Robotics and Biomimetics (ROBIO), pp. 2159-2164, IEEE, Qingdao, China, December 2016.

[10] C.-H. Chiang, "Vision-based coverage navigation for robot trash collection task," in Proceedings of the 2015 International Conference on Advanced Robotics and Intelligent Systems (ARIS), pp. 1-6, IEEE, Taipei, Taiwan, May 2015.

[11] H. Milinda and B. Madhusanka, "Mud and dirt separation method for floor cleaning robot," in Proceedings of the 2017 Moratuwa Engineering Research Conference (MERCon), pp. 316-320, IEEE, Moratuwa, Sri Lanka, May 2017.

[12] A. Grünauer, G. Halmetschlager-Funek, J. Prankl, and M. Vincze, "The power of gmms: unsupervised dirt spot detection for industrial floor cleaning robots," in Proceedings of the Annual Conference Towards Autonomous Robotic Systems, pp. 436-449, Springer, Guildford, UK, June 2017.

[13] C. Luo, S. X. Yang, X. Li, and M. Q.-H. Meng, "Neuraldynamics-driven complete area coverage navigation through cooperation of multiple mobile robots," IEEE Transactions on Industrial Electronics, vol. 64, no. 1, pp. 750-760, 2016.

[14] J. Kim, A. K. Mishra, R. Limosani et al., "Control strategies for cleaning robots in domestic applications: a comprehensive review," International Journal of Advanced Robotic Systems, vol. 16, no. 4, 2019.

[15] M. A. V. J. Muthugala, A. Vengadesh, X. Wu et al., "Expressing attention requirement of a floor cleaning robot through interactive lights," Automation in Construction, vol. 110, Article ID 103015, 2020.

[16] S. Song and S. Yamada, "Designing led lights for a robot to communicate gaze," Advanced Robotics, vol. 33, no. 7-8, pp. 360-368, 2019. 
[17] K. Zheng, G. Chen, G. Cui, Y. Chen, F. Wu, and X. Chen, "Performance metrics for coverage of cleaning robots with mocap system," in Proceedings of the International Conference on Intelligent Robotics and Applications, pp. 267-274, Springer, Wuhan, China, August 2017.

[18] M. A. V. J. Muthugala, S. M. B. P. Samarakoon, and M. R. Elara, "Tradeoff between area coverage and energy usage of a self-reconfigurable floor cleaning robot based on user preference," IEEE Access, vol. 8, pp. 76267-76275, 2020.

[19] D. Zarrouk and L. Yehezkel, "Rising star: a highly reconfigurable sprawl tuned robot," IEEE Robotics and Automation Letters, vol. 3, no. 3, pp. 1888-1895, 2018.

[20] Z. Luo, J. Shang, G. Wei, and L. Ren, "A reconfigurable hybrid wheel-track mobile robot based on watt II six-bar linkage," Mechanism and Machine Theory, vol. 128, pp. 16-32, 2018.

[21] M. Norouzi, J. V. Miro, and G. Dissanayake, "Planning stable and efficient paths for reconfigurable robots on uneven terrain," Journal of Intelligent \& Robotic Systems, vol. 87, no. 2, pp. 291-312, 2017.

[22] N. Tan, A. A. Hayat, M. R. Elara, and K. L. Wood, "A framework for taxonomy and evaluation of self-reconfigurable robotic systems," IEEE Access, vol. 8, pp. 13969-13986, 2020.

[23] V. Prabakaran, M. R. Elara, T. Pathmakumar, and S. Nansai, "htetro: a tetris inspired shape shifting floor cleaning robot," in Proceeedings of the 2017 IEEE International Conference on Robotics and Automation (ICRA), pp. 6105-6112, IEEE, Singapore, June 2017.

[24] R. Parween, A. V. Le, Y. Shi, and M. R. Elara, "System level modeling and control design of htetrakis-a polyiamond inspired self-reconfigurable floor tiling robot," IEEE Access, vol. 8, pp. 88177-88187, 2020.

[25] P. Veerajagadheswar, V. Sivanantham, M. Devarassu, and M. R. Elara, "Htetran-a polyabolo inspired self reconfigurable tiling robot," in Proceedings of the 2019 IEEE/RSJ International Conference on Intelligent Robots and Systems (IROS), pp. 4877-4884, IEEE, Singapore, June 2019.

[26] R. Parween, M. V. Heredia, M. Rayguru, R. E. Abdulkader, and M. R. Elara, "Autonomous self-reconfigurable floor cleaning robot,” IEEE Access, vol. 8, 2020.

[27] A. K. Lakshmanan, R. E. Mohan, B. Ramalingam et al., "Complete coverage path planning using reinforcement learning for tetromino based cleaning and maintenance robot," Automation in Construction, vol. 112, Article ID 103078, 2020.

[28] S. M. B. P. Samarakoon, M. A. V. J. Muthugala, A. V. Le, and M. R. Elara, "htetro-infi: a reconfigurable floor cleaning robot with infinite morphologies," IEEE Access, vol. 8, pp. 6981669828, 2020.

[29] S. M. B. P. Samarakoon, M. A. J. V. Muthugala, A. Le, and M. R. Elara, "Toward complete area coverage of a reconfigurable tiling robot by following obstacle shape," Complex and Intelligent Systems, vol. 7, 2021.

[30] M. Wainwright, "Extreme pleomorphism and the bacterial life cycle: a forgotten controversy," Perspectives in Biology and Medicine, vol. 40, no. 3, pp. 407-414, 1997.

[31] Y. Shi, M. R. Elara, A. V. Le, V. Prabakaran, and K. L. Wood, "Path tracking control of self-reconfigurable robot htetro with four differential drive units," IEEE Robotics and Automation Letters, vol. 5, no. 3, pp. 3998-4005, 2020.

[32] M. Nazarahari, E. Khanmirza, and S. Doostie, "Multi-objective multi-robot path planning in continuous environment using an enhanced genetic algorithm," Expert Systems with Applications, vol. 115, pp. 106-120, 2019.
[33] B. Vaissier, J.-P. Pernot, L. Chougrani, and P. Véron, "Genetic-algorithm based framework for lattice support structure optimization in additive manufacturing," Computer-Aided Design, vol. 110, pp. 11-23, 2019.

[34] R. J. Alattas, S. Patel, and T. M. Sobh, "Evolutionary modular robotics: survey and analysis," Journal of Intelligent \& Robotic Systems, vol. 95, no. 3-4, pp. 815-828, 2019.

[35] O. Kramer, Genetic Algorithm Essentials, Vol. 679, Springer, Berlin, Germany, 2017.

[36] S. S. Sawilowsky, "New effect size rules of thumb," Journal of Modern Applied Statistical Methods, vol. 8, no. 2, p. 26, 2009. 